\title{
Hosts and transmission of Mycobacterium ulcerans: a systematic review
}

\author{
Aboagye, I. F. \\ Department of Animal Biology and Conservation Science, University of Ghana, \\ P. O. Box LG 67, Legon-Accra, Ghana \\ Correspondence to: iaboagye@ug.edu.gh; +233249874408
}

\begin{abstract}
:
The control of Buruli ulcer (BU), a debilitating neglected tropical disease, is hampered by the inadequate understanding of the mode of transmission of its causative agent, Mycobacterium ulcerans (M. ulcerans). The DNA of $M$. ulcerans has been detected in some living organisms and non-living environmental samples of both aquatic and terrestrial sources. However, it is unclear whether the identified organisms support in vivo multiplication of the bacterium or play any role in its transmission. This paper identifies hosts of $M$. ulcerans, reviews progress made in unravelling the exact mode of transmission of $M$. ulcerans and identifies research gaps in this aspect of BU epidemiology. Using the search terms, 'niche, Mycobacterium ulcerans' and 'mode of transmission, Mycobacterium ulcerans' as well as defined inclusion criteria, information was obtained from the PubMed database and reviewed to assess their importance to the research question. Aquatic bugs of the genera Appasus and Diplonychus as well as Naucoris cimicoides and possums were identified to support in vivo multiplication of the bacterium. Bite of $M$. ulcerans contaminated Aedes notoscriptus, bite of aquatic bugs harboring or contaminated with $M$. ulcerans, and $M$. ulcerans contaminated skin-puncturing materials present in nature create opportunity for its transmission and infection. Appropriate protective measures may be useful to reduce the risk of exposure to $M$. ulcerans in $\mathrm{BU}$ endemic areas, and incorporation of trophic interactions of aquatic organisms known to support in vivo multiplication of $M$. ulcerans is needed in future research for better understanding of the spread of $M$. ulcerans in nature.
\end{abstract}

Keywords: Mycobacterium ulcerans; niche; transmission

Received May 25, 2021; Revised Jul 20, 2021; Accepted Jul 24, 2021

Copyright 2021 AJCEM Open Access. This article is licensed and distributed under the terms of the Creative Commons Attrition 4.0 International License $<$ a rel="license" href="http://creativecommons.org/licenses/by/4.0/", which permits unrestricted use, distribution and reproduction in any medium, provided credit is given to the original author(s) and the source. Editor-in-Chief: Prof. S. S. Taiwo

\section{Hôtes et transmission de Mycobacterium ulcerans: une revue systématique}

\author{
Aboagye, I. F. \\ Département de biologie animale et des sciences de la conservation, Université du Ghana, \\ Boîte postale LG 67, Legon-Accra, Ghana \\ *Correspondance à: iaboagye@ug.edu.gh; +233249874408
}

\begin{abstract}
Abstrait:
Le contrôle de l'ulcère de Buruli (UB), une maladie tropicale négligée débilitante, est entravé par la compréhension insuffisante du mode de transmission de son agent causal, Mycobacterium ulcerans (M. ulcerans). L'ADN de M. ulcerans a été détecté dans certains organismes vivants et des échantillons environnementaux non vivants de sources aquatiques et terrestres. Cependant, il n'est pas clair si les organismes identifiés favorisent la multiplication in vivo de la bactérie ou jouent un rôle dans sa transmission. Cet article identifie les hôtes de $M$. ulcerans, passe en
\end{abstract}


revue les progrès réalisés pour démêler le mode exact de transmission de $M$. ulcerans et identifie les lacunes de la recherche dans cet aspect de l'épidémiologie de I'UB. À l'aide des termes de recherche « niche, Mycobacterium ulcerans » et «mode de transmission, Mycobacterium ulcerans » ainsi que des critères d'inclusion définis, des informations ont été obtenues à partir de la base de données PubMed et examinées pour évaluer leur importance pour la question de recherche. Des punaises aquatiques des genres Appasus et Diplonychus ainsi que Naucoris cimicoides et possums ont été identifiées pour soutenir la multiplication in vivo de la bactérie. La piqûre d'Aedes notoscriptus contaminé par $M$. ulcerans, la piqûre d'insectes aquatiques hébergeant ou contaminés par $M$. ulcerans et les matériaux de perforation de la peau contaminés par $M$. ulcerans présents dans la nature créent une opportunité de transmission et d'infection. Des mesures de protection appropriées peuvent être utiles pour réduire le risque d'exposition à $M$. ulcerans dans les zones d'endémie UB, et l'incorporation d'interactions trophiques d'organismes aquatiques connus pour favoriser la multiplication in vivo de $M$. ulcerans est nécessaire dans les recherches futures pour une meilleure compréhension de la propagation de $M$. ulcerans dans la nature.

Mots clés: Mycobacterium ulcerans; niche; transmission

\section{Introduction:}

Buruli ulcer (BU) is a chronic, necrotizing and indolent disease of the skin, subcutaneous tissue and occasionally bones (1), caused by Mycobacterium ulcerans. It usually occurs in the vicinity of rural tropical wetlands, and its discovery dated back to 1897 . The disease has been reported in 33 countries globally $(2)$, mostly in tropical $(3,4)$ and subtropical regions (3). However, the largest number of cases has been reported from riverine areas in distinct regions of Benin, Côte d'Ivoire and Ghana as well as Cameroon and the Democratic Republic of Congo (3) in West and Central Africa respectively. The profound morbidity in BU victims and the devastating nature of its complications (5) have enormous adverse socioeconomic implications. This calls for more research in the grey areas of the disease, such as the mode of transmission of the bacterium, to help improve our understanding and control of the disease.

The mode of transmission of $M$. ulcerans has been a subject of investigation since 1948 (6) when the bacterium was identified as the causative agent of BU. However, the reservoir and mode(s) of transmission of the bacterium are not definitively known, posing great challenge to $\mathrm{BU}$ epidemiology. $M$. ulcerans is reported to adopt a biofilm-like structure in vitro and in vivo, and displays abundant extracellular matrix (ECM), which enhances colonization of insect vectors and mammalian hosts and confers to it increased resistance to antimicrobial agents (7). Additionally, M. ulcerans and, to a greater extent, its deoxyribonucleic acid (DNA), have been identified in various environmental samples such as inanimate materials (8-10), plants $(11,12)$, invertebrates $(13$ -16) and vertebrates $(9,17-21)$ of both aquatic and terrestrial habitats.

Two major research gaps are whether these samples support active multiplication of the bacterium and/or play any active role in its transmission. This paper identifies hosts of $M$. ulcerans, reviews progress made in the quest to unravel the exact mode(s) of transmission and identifies specific gaps that may generate interest in research in this aspect of BU epidemiology.

\section{Methodology:}

This systematic review was undertaken using the PRISMA guidelines (22) developed by the Centre for Review Dissemination (CRD). The following search terms were used to obtain information for all years from PubMed database; 'niche, Mycobacterium ulcerans' (NMU) and 'mode of transmission, Mycobacterium ulcerans' (MTMU). The searches were carried out on $2^{\text {nd }}$ September 2020 and the filters applied; abstract, free full text, full text and journal article limited the years from 2002 to 2020 and 2001 to 2020 for NMU and MTMU respectively.

The diagnosis of $\mathrm{BU}$ by the polymerase chain reaction (PCR) is based on the amplification of the insertion sequence IS2404 in the genome of $M$. ulcerans (23-25) using appropriate primers, and IS2404 PCR is considered the most sensitive method for laboratory confirmation of the disease (26). However, the detection of $M$. ulcerans from environmental samples requires confirmatory PCR targeting additional insertion sequence, IS2606, and the ketoreductase $B$ domain (KR) of $M$. ulcerans mycolactone polyketide synthase genes, to differentiate $M$. ulcerans from other environmental mycobacteria that may carry IS2404, and other non-mycolactone-producing mycobacteria (8). Therefore, studies with environmental samples having culture-confirmed $M$. ulcerans and/or PCR-positive $M$. ulcerans DNA (IS2404, KR and IS2606) (8) as well as IS2404 and $\mathrm{KR}$ with cycle thresholds $(\mathrm{Ct})$ of less than 34 and 36 for IS2404 and KR-B respectively $(8,27)$ were included in the review. The 
inclusion of IS2606 and KR of the M. ulcerans mycolactone polyketide synthase genes is based on the observation that their detection by PCR augment the specificity of IS2404 PCR for the analysis of various environmental samples (8). Other studies, including laboratory based, reporting on successful and/or proposed M. ulcerans transmission, were also included in the review. Studies that did not meet these criteria, including review articles, were excluded from the review.

\section{Results:}

\section{Number and selection of literature}

The total number of articles obtained from both searches, in PubMed database, using NMU and MTMU search terms were 58 and 24 respectively with no duplicates. Three addi- tional relevant articles were downloaded from Google Scholar. Out of the total of 85 identified articles, 57 were excluded, as they were deemed to be irrelevant to the research question after reading the titles and abstracts. After assessing full-text articles of the remaining 28 for eligibility, 15 of these were included in the review. The literature selection process is shown in Fig 1.

Sources and reservoirs of $M$. ulcerans in nature M. ulcerans and its DNA were identified from various sources including aquatic, living and inanimate sources. Table 1 describes the type and source of sample from which $M$. ulcerans and its DNA were detected as well as the detection method and key findings.

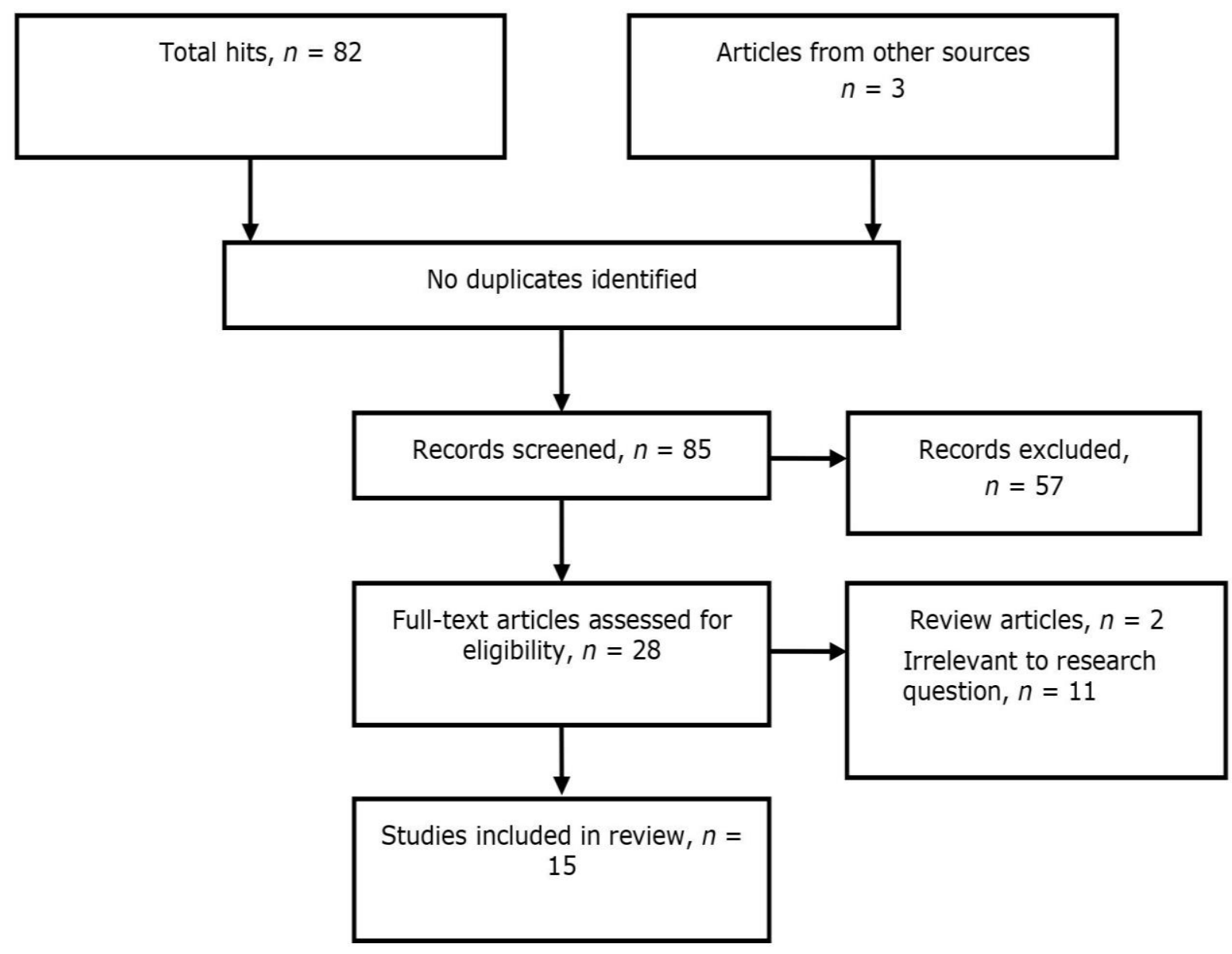

Fig 1: Flow chart of study selection process (PRISMA guide) 
Table 1: Identified sources of Mycobacterium ulcerans and its DNA (IS2404, IS2606 and KR)

\begin{tabular}{|c|c|c|}
\hline $\begin{array}{l}\text { Sample type } \\
\text { (reference) }\end{array}$ & Sample size and source of study & Detection method and result \\
\hline $\begin{array}{l}\text { Freshwater green } \\
\text { algae (11) }\end{array}$ & $\begin{array}{l}\text { Two green algae, Rhizoclonium sp. and } \\
\text { Hydrodictyon reticulatum, on mud or rock } \\
\text { surface in freshwater in BU endemic and } \\
\text { non-endemic areas, Ivory Coast. }\end{array}$ & $\begin{array}{l}\text { M. ulcerans biofilm formed on Rhizoclonium sp. } \\
\text { One positive BACTEC culture supplemented with } \\
\text { algal extract, IS2404 PCR positive on culture } \\
\text { and IS2404 PCR positive for two aquatic plant } \\
\text { samples in BU endemic area. }\end{array}$ \\
\hline Amoeba (28) & $\begin{array}{l}\text { Amoeba cultures from water }(n=13) \text {, } \\
\text { herbaceous plant biofilms }(n=90) \text { and } \\
\text { aquatic detritus samples }(n=45) \text { in and } \\
\text { around water bodies in BU endemic and } \\
\text { non-endemic communities, Ghana. }\end{array}$ & $\begin{array}{l}\text { Real-time PCR (RT-PCR) positive for IS } 2404 \text {, } \\
\text { IS } 2606 \text { and KR in } 1 \text { of } 148 \text { environmental } \\
\text { samples. IS } 2404 \text { positive for } 7 \text { out of } 166 \\
\text { amoeba cultures from } 124 \text { samples from BU } \\
\text { endemic and non-endemic communities. }\end{array}$ \\
\hline Amoeba (29) & $\begin{array}{l}\text { Free-living amoeba (FLA) cultures from } \\
\text { plant and tree trunk biofilms }(n=428), \\
\text { water }(n=53) \text {, detritus }(n=45) \text { and } \\
\text { aerosols in BU endemic and non-endemic } \\
\text { communities, Ghana. }\end{array}$ & $\begin{array}{l}\text { Quantitative PCR (qPCR) positive for IS2404 in } \\
25(4.64 \% \text { ) out of } 370 \text { FLA cultures from } 539 \\
\text { specimens, but negative for IS2606 and KR. } \\
\text { Green fluorescence protein (GFP) expressed M. } \\
\text { ulcerans in laboratory-infected Acanthamoeba } \\
\text { castellanii by flow cytometry. }\end{array}$ \\
\hline $\begin{array}{l}\text { Creeping water bugs } \\
\text { (14) }\end{array}$ & $\begin{array}{l}\text { Salivary glands of adult Naucoridae }(n= \\
80) \text { from a river in BU endemic area, } \\
\text { Ivory Coast. } \\
\text {. }\end{array}$ & $\begin{array}{l}\text { Two Culture positive for M. ulcerans and } 5 \text { of } 80 \\
\text { IS } 2404 \text { nested-PCR positive detection in } \\
\text { naturally-infected Naucoridae. Culture positive } \\
\text { strains inoculated into mice tail produced } \\
\text { inflammatory lesions with edema that were PCR } \\
\text { positive for M. ulcerans. }\end{array}$ \\
\hline $\begin{array}{l}\text { Aquatic Heteroptera } \\
\text { (30) }\end{array}$ & $\begin{array}{l}\text { Single-taxon batches }(n=283) \text { of } \\
\text { Belostomatidae, Naucoridae, Corixidae, } \\
\text { Ranatridae Nepidae, and Saliva of } \\
\text { Diplonychus sp. }(n=69) \text { from ponds } \\
\text { near villages in BU endemic area, Ivory } \\
\text { Coast. }\end{array}$ & $\begin{array}{l}\text { Real-time PCR positive for IS } 2404 \text { and KR in } 26 \\
\text { of } 283 \text { single-taxon batches of insect families } \\
\text { and } 6 \text { of } 69 \text { random saliva samples of } \\
\text { Diplonychus sp. }\end{array}$ \\
\hline Mosquitoes (16) & $\begin{array}{l}\text { Mosquitoes ( } n=11,504) \text {, mainly Aedes } \\
\text { camptorhynchus, from BU endemic area, } \\
\text { Australia. }\end{array}$ & $\begin{array}{l}\text { Of the } 11,504 \text { mosquitoes tested, } 13 \text { pools were } \\
\text { positive for IS } 2404, \text { KR and IS } 2606 \text {. VNTR locus } \\
9 \text { ( } 2 \text { positive pools) sequence identical to local } \\
\text { M. ulcerans human strain. }\end{array}$ \\
\hline Mosquitoes (31) & $\begin{array}{l}\text { Adult mosquitoes ( } n=41,797 \text { ), mainly } \\
\text { Aedes camptorhynchus, from BU endemic } \\
\text { areas, Australia. }\end{array}$ & $\begin{array}{l}\text { Real-time PCR for IS2404 ( } \pm \text { IS } 2606 \text { and KR) } \\
\text { detection rate ranged from } 1.02 \text { to } 10.80 \text { per } \\
1,000 \text { mosquitoes. Highest proportions of } M \text {. } \\
\text { ulcerans-positive mosquitoes detected in areas } \\
\text { with highest BU incidences. }\end{array}$ \\
\hline $\begin{array}{l}\text { Mosquitoes/flying } \\
\text { insects, aquatic plants, } \\
\text { invertebrate and } \\
\text { vertebrate ( } 32 \text { ) }\end{array}$ & $\begin{array}{l}\text { Flying insects ( } n=7,230) \text {, including } \\
\text { mosquito spp. ( } n=4,322), \text { Macro- } \\
\text { invertebrate and vertebrate }(n=3,377) \text {, } \\
\text { plants }((n=95) \text { from water sources near } \\
\text { villages in highly BU endemic areas, } \\
\text { Benin. }\end{array}$ & $\begin{array}{l}\text { qPCR positive for IS } 2404 \text { and KR in } 8.7 \% \\
\text { ( } 28 / 322 \text { pools) of aquatic insects including } \\
\text { water bugs, but not in mosquitoes or other } \\
\text { flying insects. Positive-PCR for } 2.1 \%(2 / 95) \\
\text { plants in the Poaceae family. }\end{array}$ \\
\hline $\begin{array}{l}\text { Mosquitoes and march } \\
\text { flies (33) }\end{array}$ & $\begin{array}{l}\text { Mosquitoes ( } n=16,900) \text { allocated to } 845 \\
\text { pools and march flies }(n=296) \text { from BU } \\
\text { endemic areas, Australia. }\end{array}$ & $\begin{array}{l}\text { Real-time PCR positive for IS2404, IS2606 and } \\
\text { KR in one pool of mosquito (Verrallina sp.) out } \\
\text { of } 845 \text { pools screened, but negative in march } \\
\text { flies. }\end{array}$ \\
\hline $\begin{array}{l}\text { Ringtail and brushtail } \\
\text { possums (9) }\end{array}$ & $\begin{array}{l}\text { Faecal samples from ringtail }(n=589) \\
\text { and brushtail }(n=250) \text { possums from BU } \\
\text { highly, low and non-endemic areas, } \\
\text { Australia. }\end{array}$ & $\begin{array}{l}\text { PCR positive for M. ulcerans DNA (IS2404, } \\
\text { IS } 2606 \text { and KR) highest in highly BU endemic } \\
\text { areas. Culture negative, but VNTR positive for } \\
\text { M. ulcerans human strain in faecal samples. }\end{array}$ \\
\hline
\end{tabular}


Inanimate materials, terrestrial and aquatic plants (9)

Ringtail and brushtail possums (34)

Bandicoot, white-tailed rats and possum (35)

Aquatic bugs (Heteroptera) (36)

Domestic animals (21)

Swabs of skin lesions $(n=25)$ out of 361 domestic animals surveyed in BU endemic areas, Benin. Swabs of skin lesions $(n=44)$ out of 397 domestic animals surveyed in BU endemic areas in Cameroon.

Domestic animals (37) Faecal samples $(n=180)$ of chickens, goats, sheep, dogs and lizards from BU endemic and non-endemic villages in Ghana.
Laboratory-confirmed (PCR \pm culture) $M$ ulcerans lesions and/or PCR-positive faeces in $16(38 \%)$ ringtail and $5(24 \%)$ brushtail possums.

Low levels of $M$. ulcerans DNA (weak positive real-time PCR signals for IS2404, IS2606 and $\mathrm{KR}$ ), but relatively higher number of positive samples in the high BU endemic area.

Culture positive for skin lesions (19), liver, spleen, mandibular lymph node (1) and skin lesions, liver, lung and small intestinal contents (1) cases. IS2404 PCR positive for faecal (14), buccal swab (7), pouch swab (3) and cloacal (1) samples, but negative for blood and urine samples.

Real-time PCR positive for IS2404, IS2606 and $\mathrm{KR}$ in 2 out of 140 bandicoot scat samples, but negative in other scat and swab samples.

qPCR positive for IS2404 and KR in 68 pools out of $616(11 \%)$ in BU endemic area, but all 80 pools negative in non-endemic area. qPCR positive for IS2404 and KR in $17.4 \%$ saliva (51/293) and tissue samples of Appasus sp. in endemic area. M. ulcerans DNA was detected in five out of seven analyzed insect families.

qPCR positive for IS2404, IS2606 and KR in 2 $(8 \%)$ external lesions of a goat and a dog out of 36 animals with lesions in Benin, but none in communities in Cameroon.

qPCR negative for $M$. ulcerans DNA targets IS2404 and KR-B.

\section{Discussion:}

\section{Ecology of M. ulcerans}

Mycobacterium ulcerans or its DNA (IS 2404, IS2606 and KR) is found associated with various aquatic and terrestrial organisms as well as inanimate materials of aquatic and terrestrial sources (Table 1 ). In BU endemic areas in particular, M. ulcerans or its DNA is found associated with freshwater green algae (11), amoeba $(28,29)$, aquatic bugs of the Order Hemiptera, including Naucoridae [creeping water bugs] $(14,30)$, Belostomatidae [giant water bugs] $(30,36)$, Corixidae [water boat- men], Ranatridae, and Nepidae [water scorpions] (30). Similar observations were made in mosquitoes $(16,31,33)$, bandicoot scat samples (35), ringtail and brushtail possums $(9,34)$, goats and dogs (21), as well as inanimate materials such as suspended solids, detritus, sediment and soil samples (9) in BU-endemic areas. Interestingly, higher levels of $M$. ulcerans DNA tend to be detected in BU-endemic areas compared with non-endemic ones.

The type of association of $M$. ulcerans with these hosts will provide insight into the maintenance and distribution of $M$. ulcerans in the environment. $M$. ulcerans is known to survive best under low oxygen tension, such as 
exist in mud in the bottom of swamps (38) where the roots of the aquatic plants, Cyperus, Panicum and Eichhornia shelter aquatic bugs (39). Crude organic extracts from two freshwater green algae, Rhizoclonium sp and Hydrodictyon reticulatum, from BU-endemic areas in tropical and temperate regions respectively, are able to stimulate the growth of $M$. ulcerans in a culture medium (11). This discovery is supported by the fact that aquatic plants are able to secrete many organic compounds, including amino acids and polysaccharides, which are used by bacteria as substrates for growth (40-42). Interestingly, genotypic analysis carried out previously (11) showed that plantassociated $M$. ulcerans had the same profile as $M$. ulcerans isolates recovered in the same region from both aquatic insects and clinical specimens. By virtue of their habitat and predatory habit, it is probable that aquatic bugs get contaminated with $M$. ulcerans or acquire it through their food chain, calling for better understanding of the ecology of these bugs.

The identification of other reservoirs and hosts that support active multiplication and shedding of $M$. ulcerans cells into the environment will be an important step in our understanding of the spread of the bacterium. $M$. ulcerans remains viable in experimentallyinfected Acanthamoeba polyphaga (28) and its IS2404 is detectable in amoeba cultures isolated from the environment $(28,29)$, suggesting that amoebae are potential natural hosts for M. ulcerans. The exclusive localization and survival of $M$. ulcerans within the salivary glands of Naucoris cimicoides (14), detection of M. ulcerans DNA in saliva of Diplonychus sp. (30), saliva and tissue samples of Appasus sp. (36), as well as the successful cultivation of $M$. ulcerans from water striders [Gerris sp.] (15), are evidence that aquatic bugs support active multiplication of $M$. ulcerans and may shed them into the environment. The common ringtail (Pseudocheirus peregrinus) and brushtail (Trichosurus vulpecula) possums may also shed viable M. ulcerans into the environment, as they are implicated as reservoirs for $M$. ulcerans (9), having had M. ulcerans PCR positive faeces and developed laboratory-confirmed M. ulcerans skin lesions.

The discovery of a biofilm sample, from water body, with similar variable number of tandem repeat (VNTR) profile to a patient sample in BU endemic community (43) suggests that the victim might have been infected following exposure to the water body. Several other environmental samples have been reported to be sources of $M$. ulcerans infection in humans. However, the possibility of BU victims shedding viable $M$. ulcerans into the environment requires investigation. The routes of spread of $M$. ulcerans and how they contribute to infection and development of $\mathrm{BU}$ should be a research priority.

\section{Transmission of M. ulcerans}

The search for the exact mode(s) of transmission of $M$. ulcerans has been challenging since its discovery as the causative agent for BU in 1948 (6). Laboratory investigations suggest that contact of mammalian skin with $M$. ulcerans does not result in infection, as mouse tails coated in $M$. ulcerans (44) and introduction of $M$. ulcerans onto skin abrasions in guinea pig models (45) are not enough to cause BU. However, the introduction of $M$. ulcerans into skin greatly facilitates infection.

Multiple proposed modes of transmission of $M$. ulcerans, including insect bite and contamination of traumatic skin sites, are documented in literature. The idea that mosquitoes may be involved in the transmission of $M$. ulcerans is premised on the association of $M$. ulcerans or its DNA with several species of mosquitoes in nature $(16,31,33)$ as well as the positive correlation between the proportion of M. ulcerans-positive mosquitoes and the number of BU cases $(16,46-48)$. The larvae of several species of mosquito remain infected with M. ulcerans throughout larval development, although the infections are not carried over into the pupae or adult mosquitoes (46). This observation suggests that mosquitoes may not serve as biological vectors for $M$. ulcerans. However, mechanical transmission of $M$. ulcerans involving blood feeding Aedes notoscriptus has been proposed (44). It appears the size of the mosquito's penetrating appendage or structure contaminated by $M$. ulcerans is important, as relatively larger $A$. notoscriptus established $\mathrm{BU}$ in mice model unlike $A$. aegypti (44). Further study involving multiple mosquito bites at $M$. ulcerans-contaminated skin surfaces, and M. ulcerans infection doses in proposed vectors, is recommended.

Mechanical transmission of $M$. ulcerans by predatory aquatic bugs, through biting (17), has strongly been proposed. The experimental infection of aquatic bugs (Naucoris cimicoides) following feeding on grubs experimentallyinfected with $M$. ulcerans and its transmission to mice through the bite of these insects (14) is documented as the first strong evidence implicating insects in the transmission of the bacte- 
rium. Belostomatidae insects of the genera Appasus (36) and Diplonychus (30) as well as Naucoris cimicoides (14) support multiplication of viable $M$. ulcerans in their salivary glands. These aquatic bugs inflict painful bites on humans, creating opportunity for the introduction of $M$. ulcerans into the skin and facilitating infection.

Trauma may be essential for the introduction of $M$. ulcerans into the skin (49), since lesions often develop at sites of skin trauma. Interestingly, mechanical transmission of $M$. ulcerans involving anthropogenic or natural skin-puncturing microtrauma has been suggested (44). This proposed method of transmission complicates the search for definitive mode of transmission of $M$. ulcerans, as any $M$. ulcerans-contaminated material capable of causing minor injuries has the potential of transmitting it. It highlights the importance of avoiding exposure to skin-puncturing microtrauma and insect bites in BU endemic areas.

\section{Understanding $M$. ulcerans spread: the way forward}

One of the key challenges in the control of $\mathrm{BU}$ is the inadequate understanding of the spread of $M$. ulcerans in nature. The contamination of various environmental samples with M. ulcerans DNA complicates the spread and mode of transmission of the bacterium. Fundamental to understanding the mode of spread of M. ulcerans in nature is the identification of factors that drive the spread.

Organisms that support in vivo multiplication of $M$. ulcerans such as aquatic hemipterans of the genera Appasus (36), Diplonychus (30), Naucoris (14) and Gerris (15) may play important role in its spread. Therefore, epidemiological studies of $M$. ulcerans that incorporate trophic interactions of such organisms may be important future research direction.

\section{Conclusion:}

This review reveals that transmission of M. ulcerans requires the introduction of viable organism into the skin of its host. Aquatic bugs of the genera Appasus and Diplonychus as well as Naucoris cimicoides support multiplication of viable $M$. ulcerans in their salivary glands. The bite of insects, such as aquatic bugs, harboring or contaminated with viable $M$. ulcerans creates opportunity for infection following the introduction of the bacterium into host skin. Similarly, skin-puncturing materials found in nature that are contaminated by $M$. ulcerans can also cause infection when these materials cause traumatic injuries to the hosts.

Although the mode of transmission of M. ulcerans is less-definitive, appropriate protective measures may reduce the risk of exposure to the bacterium in BU-endemic areas. Future research on the epidemiology of $M$. ulcerans should incorporate trophic interactions of aquatic organisms known to support in vivo multiplication of the bacterium to improve our understanding of its spread in nature.

\section{Acknowledgments:}

The author appreciates the Department of Animal Biology and Conservation Science (DABCS), University of Ghana, to which he is affiliated.

\section{References:}

1. Portaels, F., and Meyers, W. M. Buruli ulcer. In: Faber, W. R., Hay, R. J., and Naafs, B. (eds). Imported skin diseases. Elsevier Gezondheidszorg: Maarssen, The Netherlands 2006: 117-129.

2. Yotsu, R. R., Suzuki, K, Simmonds, R., et al. Buruli ulcer: a review of the current knowledge. Curr Trop Med Rep. 2018; 5: 247-256.

doi: https://doi.org/10.1007/s40475-018-0166-2

3. World Health Organization: Buruli ulcer Epidemiology; Worldwide Distribution of Buruli ulcer, 2015.

https://www.who.int/buruli/epidemiology/en/ (accessed 2 November 2020).

4. World Health Organization. Buruli ulcer - number of new cases of Buruli ulcer reported: 2018. http://apps.who.int/neglected_diseases/ntddata/b uruli/buruli.html (accessed 17 November 2020).

5. Asiedu, K., and Etuaful, S. Socioeconomic implications of Buruli ulcer in Ghana: a three-year review. Am J Trop Med Hyg. 1998; 59: 1015-1022. doi: https://doi.org/10.4269/ajtmh.1998.59.1015

6. MacCallum, P., Tolhurst, J. C., Buckle, G., et al. A new mycobacterial infection in man. J Pathol Bacteriol. 1948; 60: 93-122.

7. Marsollier, L., Brodin, P., Jackson, M., et al. Impact of Mycobacterium ulcerans biofilm on transmissibility to ecological niches and Buruli ulcer pathogenesis. PLoS Pathog. 2007; 3: 05820594. doi:10.1371/journal.ppat.0030062

8. Fyfe, J. A., Lavender, C. J., Johnson, P. D., et al. Development and application of two multiplex realtime PCR assays for the detection of Mycobacterium ulcerans in clinical and environmental samples. Appl Environ Microbiol. 2007; 73: 4733-4740. doi: 10.1128/AEM.02971-06

9. Fyfe, J. A., Lavender, C. J., Handasyde, K. A., et al. A major role for mammals in the ecology of Mycobacterium ulcerans. PLoS Negl Trop Dis. 2010; 4: e791. doi:10.1371/journal.pntd.0000791

10. Tian, R. B., Niamke, S., Tissot-Dupont, H., et al. Detection of Mycobacterium ulcerans DNA in the Environment, Ivory Coast. PLoS One. 2016; 11: e0151567.

doi:https://doi.org/10.1371/journal.pone.0151567

11. Marsollier, L., Stinear, T., Aubry, J., et al. Aquatic plants stimulate the growth of and biofilm 
formation by Mycobacterium ulcerans in axenic culture and harbor these bacteria in the environment. Appl Environ Microbiol. 2004; 70: 1097-1103. doi: 10.1128/AEM.70.2.1097-1103. 2004

12. McIntosh, M., Williamson, H., Benbow, M. E., et al. Associations between Mycobacterium ulcerans and aquatic plant communities of West Africa: implications for Buruli ulcer disease. EcoHealth. 2014; 11: 184-196. doihttps://doi.org/10.1007/s10393-013-0898-3

13. Wilson, M. D., Boakye, D. A., Mosi, L., et al. In the case of transmission of Mycobacterium ulcerans in Buruli ulcer disease, Acanthamoeba species stand accused. Ghana Medical J. 2011; 45: 31-34. doi: $10.4314 /$ gmj.v45i1.68920

14. Marsollier, L., Robert, R., Aubry, J., et al. Aquatic insects as a vector for Mycobacterium ulcerans. Appl Environ Microbiol. 2002; 68: 4623-4628. doi: 10.1128/AEM.68.9.4623-4628.2002

15. Portaels, F., Meyers, W. M., Ablordey, A., et al. First cultivation and characterization of Mycobacterium ulcerans from the environment. PLoS Negl Trop Dis. 2008; 2: e178. doi: https://doi.org/10.1371/journal.pntd.0000178

16. Johnson, P. D. R., Azuolas, J., Lavender, C. J., et al. Mycobacterium ulcerans in mosquitoes captured during outbreak of Buruli ulcer, Southeastern Australia. Emerg Infect Dis. 2007; 13: 1653-1660. doi: 10.3201/eid1311.061369

17. Portaels, F., Chemlal, K., Elsen, P., et al. Mycobacterium ulcerans in wild animals. Rev Sci Tech Off Int Epizoot. 2001; 20: 252-264. doi: $10.20506 /$ rst.20.1.1270

18. Drancourt, M., Jarlier, V., and Raoult, D. The environmental pathogen Mycobacterium ulcerans grows in amphibian cells at low temperatures. Appl Environ Microbiol. 2002; 68: 6403 - 6404. doi: 10.1128/AEM.68.12.6403-6404.2002

19. Eddyani, M., Ofori-Adjei, D., Teugels, G., et al. Potential role for fish in transmission of Mycobacterium ulcerans disease (Buruli ulcer): an environmental study. Appl Environ Microbiol. 2004 ;70: 5679-5681. doi: 10.1128/AEM.70.9.5679-5681.2004

20. van Zyl, A., Daniel, J., Wayne, J., et al. Mycobacterium ulcerans infections in two horses in south-eastern Australia. Aust Vet J. 2010; 88: 101-106. doi: 10.1111/j.1751-0813.2009.00544.x

21. Djouaka, R., Zeukeng, F., Bigoga, J. D., et al. Domestic animals infected with Mycobacterium ulcerans - implications for transmission to humans. PLoS Negl Trop Dis. 2018; 12: e0006572. doi: https://doi.org/10.1371/journal.pntd.0006572

22. Moher, D., Liberati, A., Tetzlaff, J., et al. Prisma Group. Preferred reporting items for systematic reviews and meta-analyses: The PRISMA statement. Int J Surg. 2010; 8: 336-341. doi: https://doi.org/10.1016/j.ijsu.2010.02.007

23. Stinear, T., Ross, B. C., Davies, J. K., et al. Identification and characterization of IS2404 and IS2606: two distinct repeated sequences for detection of Mycobacterium ulcerans by PCR. J Clin Microbiol. 1999; 37: 1018-1023 doi: $10.1128 / J C M .37 .4 .1018-1023.1999$

24. Stinear, T. P., Seemann, T., Pidot, S., et al. Reductive evolution and niche adaptation inferred from the genome of Mycobacterium ulcerans, the causative agent of Buruli ulcer. Genome Res. 2007; 17: 192-200. doi:10.1101/gr.5942807

25. Eddyani, M., Sopoh, G. E., Ayelo, G., et al. Diagnostic accuracy of clinical and microbiological signs in patients with skin lesions resembling Buruli ulcer in an endemic region. Clin Infect Dis. 2018; 67: 827-34. doi: 10.1093/cid/ciy197

26. Beissner, M., Herbinger, K., and Bretzel, G. Laboratory diagnosis of Buruli ulcer disease. Future Microbiol. 2010; 5: 363-370. doi: $10.2217 / F M B .10 .3$

27. Garchitorena, A., Roche, B., Kamgang, R., et al. Mycobacterium ulcerans ecological dynamics and its association with freshwater ecosystems and aquatic communities: results from a 12-month environmental survey in Cameroon. PLoS Negl Trop Dis. 2014; 8: e2879. doi: 10.1371/journal.pntd.0002879

28. Gryseels, S., Amissah, D., Durnez, L., et al. Amoebae as potential environmental hosts for Mycobacterium ulcerans and other mycobacteria, but doubtful actors in Buruli ulcer epidemiology. PLoS Negl Trop Dis. 2012; 6: e1764. doi: 10.1371/journal.pntd.0001764

29. Amissah, N. A., Gryseels, S., Tobias, N. J., et al. Investigating the role of free-living amoebae as a reservoir for Mycobacterium ulcerans. PLoS Negl Trop Dis. 2014; 8: e3148. doi: 10.1371 /journal.pntd.0003148

30. Konan, K. L., Doannio, J. M., Coulibaly, N. G., et al. Detection of the IS2404 insertion sequence and ketoreductase produced by Mycobacterium ulcerans in the aquatic Heteroptera in the health districts of Dabou and Tiassalé in Côte d'Ivoire. Méd Santé Trop. 2015; 25: 44-51. doi: $10.1684 / \mathrm{mst} .2014 .0363$

31. Lavender, C. J., Fyfe, J. A., Azuolas, J., et al. Risk of Buruli ulcer and detection of Mycobacterium ulcerans in mosquitoes in southeastern Australia. PLoS Negl Trop Dis. 2011; 5: e1305. doi: 10.1371 /journal.pntd.0001305

32. Zogo, B., Djenontin, A., Carolan, K., et al. A field study in Benin to investigate the role of mosquitoes and other flying insects in the ecology of Mycobacterium ulcerans. PLoS Negl Trop Dis. 2015; 9: e0003941. doi: 10.1371/journal.pntd.0003941

33. Singh, A., McBride, W. J. H., Govan, B., et al. A survey on Mycobacterium ulcerans in Mosquitoes and March flies captured from endemic areas of Northern Queensland, Australia. PLoS Negl Trop Dis. 2019; 13: e0006745.

doi: https://doi.org/10.1371/journal.pntd.0006745

34. O'Brien, C. R., Handasyde, K. A., Hibble, J., et al. Clinical, microbiological and pathological findings of Mycobacterium ulcerans infection in three Australian Possum species. PLoS Negl Trop Dis. 2014; 8: e2666. doi: 10.1371/journal.pntd.0002666

35. Singh, A., McBride, J. H. W., Govan, B., et al. Survey of local fauna from endemic areas of northern Queensland, Australia for the presence of Mycobacterium ulcerans. Int $\mathrm{J}$ Mycobacteriol. 2019; 8: 48-52. doi: 10.4103/ijmy.ijmy_168_18

36. Marion, E., Eyangoh, S., Yeramian, E., et al. Seasonal and regional dynamics of $M$. ulcerans transmission in environmental context: decipherring the role of water bugs as hosts and vectors. PLoS Negl Trop Dis. 2010; 4: e731. doi: 10.1371 /journal pntd.0000731

37. Tobias, N. J., Ammisah, N. A., Ahortor, E. K., et al. Snapshot fecal survey of domestic animals in rural Ghana for Mycobacterium ulcerans. Peer]. 2016; 4: e2065. doi: 10.7717/peerj.2065

38. Palomino, J. C., Obiang, A. M., Realini, L., et al. Effect of oxygen on growth of Mycobacterium ulcerans in the BACTEC system. J Clin Microbiol. 1998; 36: 3420-3422

doi: $10.1128 / J C M .36 .11 .3420-3422.1998$ 
39. Portaels, F., Elsen, P., Guimaraes-Peres, A., et al. Insects in the transmission of Mycobacterium ulcerans infection (Buruli ulcer). Lancet. 1999; 353: 986. doi: https://doi.org/10.1016/S01406736(98)05177-0

40. Moore, B. G. Extracellular polysaccharides of algae: effects on life-support system. Science. 1964; 145: 586-587.

doi: $10.1126 /$ science. 145.3632 .586

41. Jensen, L. M. Phytoplankton release of extracellular organic carbon, molecular weight composition and bacterial assimilation. Mar Ecol Prog Ser. 1983; 11: 39-48. doi: http://www.jstor.org/stable/24815065

42. Maurin, N., Amblard, C., and Bourdier, G. Phytoplanktonic excretion and bacterial reassimilation in an oligomesotrophic lake: molecular weight fractionation. J Plankton Res. 1997; 19: 1045-1068.

doi: https://doi.org/10.1093/plankt/19.8.1045

43. Narh, C. A., Mosi, L., Quaye, C., et al. Source tracking Mycobacterium ulcerans infections in the Ashanti region, Ghana. PLoS Negl Trop Dis. 2015; 9: e0003437. doi: https://doi.org/10.1371/journal.pntd.0003437

44. Wallace, J. R., Mangas, K. M., Porter, J. L., et al. Mycobacterium ulcerans low infectious dose and mechanical transmission support insect bites and puncturing injuries in the spread of Buruli ulcer. PLoS Negl Trop Dis. 2017; 11: e0005553. doi:https://doi. org/10.1371/journal.pntd.0005553

45. Williamson, H. R., Mosi, L., Donnell, R., et al. Mycobacterium ulcerans fails to infect through skin abrasions in a guinea pig infection model: implications for transmission. PLoS Negl Trop Dis. 2014; 8: e2770.

doi: https://doi.org/10.1371/journal.pntd.0002770

46. Wallace, J. R., Gordon, M. C., Hartsell, L., et al. Int eraction of Mycobacterium ulcerans with mosquito species: implications for transmission and trophic relationships. Appl Environ Microbiol. 2010; 76: 6215-6222

doi: $10.1128 / A E M .00340-10$

47. Johnson, P. D., and Lavender, C. J. Correlation between Buruli ulcer and vector-borne notifiable diseases, Victoria, Australia. Emerg Infect Dis. 2009; 15: 614-615. doi: 10.3201/eid1504.081162

48. Lavender, C. J., and Fyfe, J. A. Direct detection of Mycobacterium ulcerans in clinical specimens and environmental samples. Methods Mol Biol. 2013; 943: $201-216$.

doi: $10.1007 / 978-1-60327-353-4 \_13$.

49. Meyers, W. M., Shelly, W. M., Connor, D. H., et al. Human Mycobacterium ulcerans infections developing at sites of trauma to skin. Am J Trop Med Hyg. 1974; 23: 919-923.

doi: https://doi.org/10.4269/ajtmh.1974.23.919 